Sección
Internacional 



\title{
La libertad de opinión y expresión en la Observación General No. 34 del Comité de Derechos Humanos del PIDCP
}

Este artículo se encuentra disponible para su descarga gratuita en www.anuariocdh.uchile.cl

\begin{abstract}
Simón Conforti
Estudiante de abogacía, Facultad de Derecho de la Universidad Nacional del Centro de la Provincia de Buenos Aires (UNICEN), Argentina. Integra como investigador alumno el Centro de Estudios en Derechos Humanos (CEDH). simonconforti@hotmail.com
\end{abstract}

\section{RESUMEN}

El Comité de Derechos Humanos emitió en 2011 la Observación General No. 34 sobre el derecho a la libertad de opinión y de expresión. Este artículo explora el contenido de esta resolución, evaluando primero la forma en que el Comité interpreta la esfera positiva de la libertad de expresión, para luego analizar en detalle el tratamiento que se le da a la esfera negativa o restricciones legítimas a este derecho -específicamente, la noción de ley utilizada por el Comité, así como el alcance de los conceptos de seguridad nacional, orden público y moral pública. A partir de los debates llevados a cabo durante la discusión de esta resolución, y comparando los estándares adoptados con aquellos establecidos en otros sistemas de protección de derechos humanos -en particular, el sistema interamericano- se concluye que esta nueva Observación General no tiene un carácter vanguardista, sino que reafirma los estándares ya asentados sobre libertad de expresión en el sistema universal de protección de los derechos humanos.

Palabras clave: Comité de Derechos Humanos Libertad de expresión - Restricciones - Seguridad nacional - Orden público - Moral pública

\section{SUMMARY}

In 2011 the Human Rights Committee brought out General Comment No. 34 on the right to freedom of opinion and expression. This article explores the content of this document by first evaluating the way in which the Committee interprets the positive sphere of freedom of expression to then analyze in detail the way in which the negative sphere (or legitimate restrictions of this right) is dealt with -specifically the concept of law used by the Committee along with the content of the concepts of national security, public order and public morals. Taking into account the debates held during the discussion of this General Comment and comparing the standards it sets with those set by other human rights protection systems - particularly the Inter-American system- the article concludes that this new General Comment does not push any boundaries but rather it reaffirms the existing standards on freedom of expression that have already been set in the universal human rights protection system.

Key words: Human Rights Committee - Freedom of expression - Restrictions - National security Public order - Public morals

\section{Introducción}

El 21 de julio de 2011 el Comité de Derechos Humanos (en adelante "el Comité") emitió su Observación General No. 34 (en adelante "la Observación General", "la Observación" o "la OG $34^{\prime \prime}$ ) sobre el derecho a la libertad de opinión y de expresión garantizado en el artículo 19 del 
Pacto Internacional de Derechos Civiles y Políticos ${ }^{1}$ (en adelante "el Pacto" o "el PIDCP"), con el objetivo de brindar, principalmente a los Estados, herramientas que ayuden a la interpretación de dicho artículo². En este sentido, el texto elaborado por el Comité "es una respuesta integral a las numerosas peticiones de los legisladores, jueces, fiscales, abogados, defensores de derechos humanos y periodistas, pidiendo una aclaración sobre muchos de los temas cubiertos por los derechos a la libertad de expresión y de opinión" ${ }^{3}$. Incluso Michael O'Flaherty, miembro del Comité y principal redactor de la observación general, dijo que esta "[e]s una fuerte reafirmación de la importancia fundamental de todos los derechos humanos y de la libertad de expresión, estableciendo parámetros muy estrictos en el derecho a restringirla por parte de los Estados" ${ }^{\prime 4}$.

Otra de las ventajas de contar con una observación general sobre libertad de expresión es la integración de distintos conceptos sobre el tema elaborados en observaciones finales, peticiones individuales, etc.; de esta manera el Comité puede rescatar ciertas apreciaciones pasadas que considera especialmente vigentes en la realidad actual.

En este artículo analizaremos algunas secciones de la OG 34, mostrando que no se trata de una resolución vanguardista, sino que reafirma los estándares que existen actualmente sobre libertad de expresión, incluso a veces de forma bastante tímida. Con todo, a nuestro juicio esta resolución del Comité de Derechos Humanos también muestra el grado de consenso que se ha alcanzado a nivel universal sobre algunas garantías mínimas relativas a la protección del derecho a la libertad de opinión y expresión, y en este sentido puede valorarse positivamente. Para ello, analizaremos en una primera sección la forma en que el Comité concibe la esfera positiva de la libertad de opinión y expresión, para en una segunda parte revisar con más detalle el sistema de restricciones que dicho órgano considera legítimo aplicar a este derecho.

\section{La esfera positiva de la libertad de opinión y expresión}

El Comité parte de la consideración de que el derecho a la libertad de opinión y expresión es la piedra angular de todas las sociedades libres y democráticas y una base del pleno disfrute de los demás derechos humanos. Este reconocimiento apriorístico muestra el grado de avance que ha tenido en los últimos años este derecho, pudiendo llegar hoy en día a ser así calificado, sin necesidad de recordar todos los fundamentos en los que se justifica. En este orden de ideas se menciona también su importancia para lograr los principios de transparencia y rendición de cuentas, requisitos esenciales para la promoción y protección de los derechos humanos.

1 Comité de Derechos Humanos. Observación General No. 34. Artículo 19. Libertad de opinión y libertad de expresión. 21 de julio de 2011. CCPR/C/GC/34. Esta Observación reemplaza a la Observación General No. 10 (Comité de Derechos Humanos. Observación General No. 10. Artículo 19. Libertad de opinión. 29 de junio de 1983. U.N. Doc. HRI/GEN/1/ Rev.7 at 150 1983).

2 El artículo 19 del Pacto Internacional de Derechos Civiles y Políticos establece que "1. Nadie podrá ser molestado a causa de sus opiniones. 2. Toda persona tiene derecho a la libertad de expresión; este derecho comprende la libertad de buscar, recibir y difundir informaciones e ideas de toda índole, sin consideración de fronteras, ya sea oralmente, por escrito o en forma impresa o artística, o por cualquier otro procedimiento de su elección. 3. El ejercicio del derecho previsto en el párrafo 2 de este artículo entraña deberes y responsabilidades especiales. Por consiguiente, puede estar sujeto a ciertas restricciones, que deberán, sin embargo, estar expresamente fijadas por la ley y ser necesarias para: a) Asegurar el respeto a los derechos o a la reputación de los demás; b) La protección de la seguridad nacional, el orden público o la salud o la moral públicas".

3 United Nations Office at Geneva (UNOG). "Human Rights Committee continues discussion of draft General Comment on the right to freedom of expression". Comunicado de prensa. 20 de julio de 2011 [en línea] <http://www.unog.ch/ unog/website/news_media.nsf/(httpNewsByYear_en)/2D22776D28A2C443C12578D3005A834D?OpenDocument> [consulta: 15 marzo 2012]. Traducción propia.

4 Ídem. 
Siguiendo a la Observación General No. $31^{5}$, se reafirma que la obligación de respetar el derecho a la libertad de expresión implica la responsabilidad de todos los poderes del Estado (ejecutivo, legislativo y judicial) y de otras entidades u organismos en los que el Estado tenga participación, incluyendo en ciertos casos a instituciones semiestatales. Además, se refiere a la obligación de garantía a nivel interno que implica proteger a las personas de los actos de privados que podrían impedir el goce de este derecho.

Estas afirmaciones se refuerzan con la incompatibilidad de reserva del artículo 19, inc. 1, estando prohibida, también en opinión del Comité, la suspensión del derecho a la libertad de opinión que se deriva del mencionado inciso. En este sentido, este derecho está protegido en todas sus formas, incluso en lo relativo al derecho a expresar o no expresar una opinión ${ }^{6}$. Respecto de la libertad de expresión deja a salvo la posibilidad de una reserva sólo a los elementos particulares del artículo 19, inc. 2.

En la Observación, se hace referencia a tres ámbitos relacionados con la esfera positiva de la libertad de expresión y que son de particular interés: los medios de comunicación, el acceso a la información pública y la relación con los derechos políticos.

En relación al primer punto, se debe destacar el carácter libre e independiente que deben tener los medios, acentuándose su función de conectar a ciudadanos, candidatos y representantes (y viceversa). A esto se agrega la pluralidad de medios como una forma de proteger los derechos de los usuarios. De esta manera, la libertad de expresión aparece configurada no solo como un derecho individual, sino también dentro de un plano colectivo donde juega una importante función social. En este orden de ideas, vemos un reconocimiento a los nuevos medios de comunicación como actores fundamentales del cambio en las prácticas de comunicación de todo el mundo, destacando que debe fomentarse su independencia y asegurarse que todos puedan acceder a ellos. El surgimiento y afianzamiento de estos nuevos canales de comunicación refleja también una visión crítica respecto a la confianza en los intermediarios de los medios tradicionales. Este reconocimiento estuvo muy presente durante las discusiones del proyecto de Observación General. Es así que "[l]os miembros del Comité dijeron que no podían abstenerse de hacer frente a esta área sólo porque la tecnología cambia constantemente. No podían esperar a la jurisprudencia para ponerse al día con la tecnología ya que los medios de comunicación impresos, audiovisuales y en la web estaban convergiendo [...] un miembro del Comité dijo que si veía a los levantamientos en el Medio Oriente y África del Norte en el último año, que fueron impulsados en gran parte por los nuevos medios, el Comité tiene que abordar esta área"7.

Respecto al acceso a la información pública, se puede destacar que en la OG 34 se establecen estándares mínimos sobre qué debe entenderse por "organismos públicos", aclarando que más allá que un órgano no posea la denominación de "estatal" será abarcado por la norma si está llevando a cabo funciones públicas ${ }^{8}$. Esto se complementa con el deber de los Estados Parte de poner la información de interés público en dominio público; lo que permite que la misma circule y pueda ser evaluada críticamente. En el sistema interamericano de protección de los derechos humanos esto mismo es vinculado con la dimensión social de la libertad de expresión y ha sido hondamente

\footnotetext{
5 Comité de Derechos Humanos. Observación General No. 31. Naturaleza de la obligación jurídica general impuesta a los Estados Parte en el Pacto. 29 de marzo de 2004. CCPR/C/21/Rev.1/Add.13.

6 Comité de Derechos Humanos. Observación General No. 34, op. cit., párr. 9.

UNOG. "Human Rights Committee continues discussion of draft General Comment...", op. cit. Traducción propia.

8 Comité de Derechos Humanos. Observación General No. 34, op. cit., párr. 18.
} 
tratado ${ }^{9}$. En cuanto a la libertad de expresión y los derechos políticos es el apartado con menor tratamiento; quizás porque la orientación de los Estados Parte ya está dada por la OG $25^{10}$.

\section{La restricción a la libertad de opinión y expresión}

El párrafo 3 del artículo 19 señala expresamente que el ejercicio del derecho a la libertad de expresión entraña deberes y responsabilidades especiales, y por ello puede estar sujeto a ciertas restricciones. En este sentido veremos los requisitos que deben cumplir las restricciones, las razones en las cuales se pueden afirmar, los criterios que deben cumplir y finalmente un breve repaso por algunos casos particulares.

\subsection{Restricción establecida por ley}

Como punto inicial se reconoce la posibilidad de establecer por "ley" restricciones a la libertad de expresión, fundada en las responsabilidades que entraña su ejercicio. El término leyes no es objeto de definición semántica, más bien se describen cualidades particulares que deben tener esas leyes, como la formulación precisa y accesible. Durante el debate se contempló que "quizás el Comité debería ser explícito en que cuando se refiere a norma legales quiere decir actos adoptados por órganos de cuerpos legislativos" ${ }^{11}$. Sin embargo, esta idea no fue recogida en el entendimiento de que "era importante no ser demasiado explícito, ya que podría limitar la lista de leyes subordinadas que se utilizan a menudo para limitar la libertad de expresión, incluyendo los reglamentos locales, las leyes tradicionales o consuetudinarias y de la ley religiosa"12, a la vez que otro experto señaló que "en algunos países los sistemas jurídicos eran ricos y variados, y los conceptos de ley informal, derecho consuetudinario, o ley religiosa no eran conceptos que no han creado confusión a lo que el uso de ese lenguaje se refiere"13. Respecto a este punto, nos parece que en la OG 34 se podrían haber recogido los avances de la determinación de la expresión leyes, que en el sistema interamericano fue hecha hace ya 25 años y que brinda parámetros para interpretar el término en cuestión más allá de las distintas acepciones que pueda tener en cada orden jurídico particular ${ }^{14}$. Esta falta de definición puede producir que los países restrinjan la libertad de expresión por vías que formalmente aparentan ser leyes, pero que de acuerdo a los criterios democráticos no lo son; entre los que podemos ubicar por ejemplo, a los decretos y las resoluciones administrativas, entre otras.

9 Ver Corte IDH. La Colegiación Obligatoria de Periodistas. Opinión Consultiva OC-5/85 del 13 de noviembre de 1985. Serie A No. 5; Corte IDH. Caso "La Última Tentación de Cristo" (Olmedo Bustos y otros) vs. Chile. Fondo, Reparaciones y Costas. Sentencia de 5 de febrero de 2001. Serie C No. 73; Corte IDH. Caso Gomes Lund y otros (Guerrilha do Araguaia) vs. Brasil. Excepciones Preliminares, Fondo, Reparaciones y Costas. Sentencia de 24 de noviembre de 2010. Serie C No. 219 y Corte IDH. Caso Fontevecchia y D'Amico vs. Argentina. Fondo, Reparaciones y Costas. Sentencia de 29 de noviembre de 2011. Serie C No. 238.

10 Comité de Derechos Humanos. Observación General No. 25. Artículo 25. La participación en los asuntos públicos y el derecho de voto. 17 de julio de 1996. CCPR/C/21/Rev.1/Add.7.

11 UNOG. "Human Rights Committee discusses draft General Comment on the right to freedom of expression". Comunicado de Prensa. 19 de julio de 2011. [en línea] <http://www.unog.ch/unog/website/news_media.nsf/(httpNewsByYear_en)/0 2E389A5A86CACAAC12578D2003EEDA1?OpenDocument> [consulta: 15 marzo 2012]. Traducción propia.

12 Ídem.

13 Ídem.

14 Corte IDH. La expresión "leyes" en el artículo 30 de la Convención Americana de Derechos Humanos. Opinión Consultiva OC-6/86 del 9 de mayo de 1986, Serie A No. 6, párrs. 26-29. 


\subsection{Las razones o motivos legítimos para las restricciones}

Un tratamiento importante reciben las razones específicas que pueden suponer una restricción a la libertad de expresión, establecidas en los puntos a y b del inciso 3 del artículo 19 (Cfr. supra nota 2) las que se refieren a dos áreas específicas. Por un lado, la que busca asegurar el respeto a los derechos o a la reputación de los demás, entendiendo a "derechos" como aquellos consagrados tanto en el Pacto como en otros organismos de derechos humanos y entendiendo a "demás", en forma individual o grupal.

Por otro lado, el segundo motivo legítimo es la protección de la seguridad nacional, el orden público o la salud o la moral públicas. Estos tres conceptos deben ser valorados cuidadosamente, ya que su vaguedad puede suponer su utilización extensiva para restringir la libertad de expresión. Analizaremos a continuación cada uno de ellos.

En primer lugar, en razón de la seguridad nacional pueden justificarse restricciones como las leyes sobre traición y disposiciones similares, y se debe prestar atención para que éstas no se vuelvan una excusa en la persecución de periodistas, investigadores, etc. También se debe procurar evitar utilizar este fundamento en las disposiciones relativas al sector comercial, la banca y el progreso científico. Sin embargo, los Estados Parte presentaron sugerencias y comentarios sobre este tema diciendo que no podían aceptar la redacción utilizada en el proyecto, "ya que representaría una prohibición total de estas leyes y reconoció que podría haber ciertas circunstancias en las que estaba permitido para los Estados prohibir ciertas formas de expresión para proteger la seguridad nacional". Bajo este panorama, algunos miembros del Comité anticiparon problemas "por temor a que este término requiera ahora de una definición de seguridad nacional y esto sería una tarea muy difícil"15. Ante esto se evitó una definición del término y el Comité se refirió a casos particulares a modo de ejemplo. Por nuestra parte, consideramos que esta restricción basada en la seguridad nacional debe aplicarse únicamente en los casos más graves, cuando exista una amenaza política o militar directa contra todo el país ${ }^{16}$. Así para el Relator de Naciones Unidas para la Libertad de Expresión, "el objetivo de proteger la seguridad nacional, perfectamente legítimo, no debe ser invocado a la ligera por los gobiernos para justificar infracciones del derecho a la libertad de expresión que serían innecesarias e inadmisibles porque no satisfacen la finalidad declarada" ${ }^{\prime 7}$.

Respecto del mantenimiento del orden público, no se abordó tampoco una definición sobre el término debido a la preocupación de si "se trataba de un camino que se debería explorar dentro de los límites de este proyecto de Observación General"18. En nuestra opinión, esta situación representaba un desafío importante para el Comité, ya que la ambigüedad semántica de la expresión en cuestión constituye un difícil problema que ha originado diversas interpretaciones. Por ejemplo, la Corte Interamericana de Derechos Humanos (Corte IDH) ha señalado que "una acepción posible del orden público dentro del marco de la Convención [Americana de Derechos Humanos] hace referencia a las condiciones que aseguran el funcionamiento armónico y normal

15 UNOG. "Human Rights Committee discusses draft...", op. cit.

16 Ver el Principio No. 2 de Los Principios de Johannesburgo sobre la Seguridad Nacional, la Libertad de Expresión y el Acceso a la Información. Londres: ARTíCULO 19, 1996 (Ed. Español: 2005).

17 Comisión de Derechos Humanos. Consejo Económico y Social. Cuestión de los derechos humanos de todas las personas sometidas a cualquier forma de detención o prisión. Promoción y protección del derecho a la libertad de opinión y de expresión. Informe del Relator Especial, Sr. Abid Hussain presentado en cumplimiento de la resolución 1993/45 de la Comisión de Derechos Humanos. 14 de diciembre de 1994. E/CN.4/1995/32, párr. 51.

18 UNOG. "Human Rights Committee adopts General Comment on the right to freedom of expression". Comunicado de Prensa. 21 de julio de 2010. [en línea] <http://www.unog.ch/unog/website/news_media.nsf/(httpNewsByYear_en)/E4D 729D3E6E0CCD5C12578D4004CF6C0?OpenDocument> [consulta: 15 marzo 2012]. Traducción propia. 
de las instituciones sobre la base de un sistema coherente de valores y principios"19. También el Relator de Naciones Unidas para la Libertad de Expresión estima que este concepto incluye "la defensa del orden y prohibición del delito", así como los "principios fundamentales universalmente reconocidos en los que se basa una sociedad democrática y que permiten el respeto de los derechos humanos" 20 . Posiblemente el Comité podría haber recogido estas interpretaciones para aportar por su parte mayores precisiones, así como nuevos enfoques a la luz del contexto actual.

En tercer lugar, la moral pública es el último motivo establecido en el punto b del inciso 3 del artículo 19, y la vaguedad propia del término es reconocida en el párrafo 32 de la OG 34, donde se propone además contemplar las diversas tradiciones de las que deriva la moral. Los expertos que participaron de la discusión de la OG 34 se refirieron a que la Observación General No. $22^{21}$ del Comité dice que la moral difiere de una sociedad a otra, por lo que algunos de ellos estaban preocupados de que, al referirse al vocablo en el proyecto, se perjudicara la libertad de expresión, ya que los Estados podrían utilizar este lenguaje como una forma de socavar el derecho sobre la base de las normas culturales o sociales.

En definitiva, la reticencia mostrada por el Comité en abordar la determinación formal de estos tres conceptos -seguridad nacional, orden público y moral pública-, produce que sus palabras pierdan contundencia. Esto se debe posiblemente a que el Comité busca un compromiso político respecto a aquellos países con menos inclinación a adoptar estándares fuertes de protección en cuanto a la libertad de expresión.

\subsection{La necesidad y proporcionalidad de las restricciones}

El requisito de "necesidad" se refiere a la exigencia de utilizar el medio menos restrictivo de la libertad de expresión y el de "proporcionalidad" como la correlación con el interés que desea proteger. El Comité define estos requisitos mediante ejemplos; sin embargo, podemos completar la definición recurriendo a distintas fuentes, en particular la Corte Europea de Derechos Humanos. Dicho tribunal, al interpretar el artículo 10 de la Convención Europea de Derechos Humanos concluyó que "necesarias", sin ser sinónimo de "indispensables", implica la existencia de una "necesidad social imperiosa" y que para que una restricción sea "necesaria" no es suficiente demostrar que sea "útil", "razonable" u "oportuna"22. Esta conclusión, que es igualmente aplicable a la Convención Americana ${ }^{23}$, sugiere que la "necesidad" y, por ende, la legitimidad de las restricciones a la libertad de expresión dependerá de que estén orientadas a satisfacer un interés público imperativo. Es tarea del Estado probar la existencia de los mencionados elementos, estableciendo una relación directa y conexión inmediata entre la expresión y la amenaza; y esto debe ser valorado tanto en la ley que define la restricción como en la aplicación al caso concreto. Esto quiere decir que no basta que la formulación legal sea acertada, sino que también su aplicación

19 Corte IDH. La Colegiación Obligatoria de Periodistas, op. cit., párr. 64.

20 Comisión de Derechos Humanos. Consejo Económico y Social. Cuestión de los derechos humanos de todas las personas..., op. cit., párr. 52.

21 Comité de Derechos Humanos. Observación General No.22. Artículo 18. El derecho a la libertad de pensamiento, conciencia y religión. 30 de julio de 1993. CPR/C/21/Rev.1/Add.4.

22 European Court of Human Rights (ECHR). The Sunday Times v. The United Kingdom. Judgment of 26 April 1979. Application No. 6538/74, párr. 59; Barthold v. Germany. Judgment of 25 March 1985. Application No. 8734/79, párr. 59; Éditions Plon v. France. Judgment of 18 May 2004. Application No. 58148/00, párr. 42 y MGN Limited v. The United Kingdom. Judgment of 18 January 2011. Application No. 39401/04, párr. 139.

23 Corte IDH. La Colegiación Obligatoria de Periodistas, op. cit.; Corte IDH. Caso Fontevecchia y D’Amico vs. Argentina, op. cit., párr. 54. 
judicial o administrativa no debe formar prácticas que desvirtúen estos estándares ${ }^{24}$. La Observación deja en claro que sobre este punto el Estado no posee "margen de apreciación" y al decir esto se refiere de forma directa a la "teoría del margen de apreciación" que ha utilizado recurrentemente la Corte Europea de Derechos Humanos ${ }^{25}$. Según esta teoría, la Corte Europea considera que las autoridades estatales son las que mejor podrán evaluar si una medida restrictiva es adecuada o no dentro de su contexto social y conforme con las condiciones particulares allí imperantes. La diferencia que marca la Observación General No. 34 radica en su rechazo a esta teoría a la hora de restringir la libertad de expresión. Esto significa que frente al Comité, el Estado debe demostrar de manera específica la naturaleza precisa de la amenaza en cualquiera de los motivos que han causado la restricción. La aplicación de las restricciones permisibles debe ser coherente con los demás derechos garantizados en el Pacto y con los principios fundamentales de igualdad y no discriminación; y más allá de la evaluación del Estado, lo que el Comité determinará es si se violó o no la obligación asumida. Esta característica ha sido utilizada por el Comité en los documentos anteriores y enfatiza el papel que se le da a la determinación del principio de necesidad. Es entendible a la luz de esta explicación que el Sr. O'Flaherty destaque la importancia de mantener la frase "margen de apreciación", debido a que ha sido una de las cosas que distingue al Comité de Derechos Humanos de la Corte Europea. Un ejemplo de ello es que mientras la Corte Europea ha dicho que "estando en contacto directo y permanente con las fuerzas vitales del país, las autoridades nacionales están en principio mejor situadas que una jurisdicción internacional para evaluar las necesidades y el contexto local"26; el Comité se ha apartado de esta interpretación afirmando que "más allá de las justificaciones que esgrima el Estado, toda medida cuyo efecto equivalga a negar el derecho no es compatible con las obligaciones establecidas en el pacto" 27.

\subsection{Algunas áreas específicas de restricciones}

Finalmente llegamos al tratamiento de áreas específicas y limitaciones en las posibilidades, formas y extensión de las restricciones. El Comité analizó:

i) Las expresiones concernientes a figuras públicas en el ámbito político y en el ámbito de las instituciones públicas;

ii) Los medios de comunicación, avances en las comunicaciones y los marcos regulatorios;

iii) La libertad especial de periodistas y comunicadores (en sentido amplio) y protección de sus fuentes.

Consideramos que es importante realizar un estudio detallado del tratamiento de cada una de estas situaciones, lo que no se puede realizar en este artículo por limitaciones de espacio, sin embargo, podemos mencionar cuestiones particularmente importantes que pueden servir como disparadores para acudir a la OG 34.

24 Ver Comité de Derechos Humanos. Observación General No. 27. Artículo 12. Libertad de circulación. 2 de noviembre de 1999. CCPR/C/21/Rev.1/Add.9 párr. 14. Véase también Comité de Derechos Humanos. Marques de Morais vs. Angola. Comunicación No. 1128/2002. Dictamen de 29 de marzo de 2005. CCPR/C/83/D/1128/2002; y Coleman vs. Australia. Comunicación No. 1157/2003. Dictamen de 17 de julio de 2006. CCPR/C/87/D/1157/2003.

25 GARCÍA ROCA, Javier. "La muy discrecional doctrina del margen de apreciación nacional según el Tribunal Europeo de Derechos Humanos: soberanía e integración". Teoría y Realidad Constitucional No. 20, UNED, 2007, p. 124.

26 ECHR. Buckley v. The United Kingdom. Judgment of 25 September 1996. Application No. 20348/92, párr. 75.

27 Comité de Derechos Humanos. Caso Ilmari Länsman y otros vs. Finlandia. Comunicación No. 511/1992. Dictamen de 14 de octubre de 1993. CCPR/C/52/D/511/1992, párr. 10. 
Sobre el primer punto se destaca la afirmación de que el mero hecho de que las formas de expresión sean consideradas como un insulto a una figura pública no es suficiente para justificar la imposición de sanciones, justamente porque la identidad de la persona que ha sido impugnada no puede ser la base que justifica la restricción ${ }^{28}$.

Respecto a los medios de comunicación se destaca su función de promover la transparencia y rendición de cuentas, debiendo establecerse requisitos razonables, objetivos claros, transparentes y no discriminatorios en el otorgamiento de las licencias ${ }^{29}$; permitiendo justamente el acceso equitativos a todos los sectores (público, comercial y comunitario). En nuestra opinión, esto debe ser complementado con el objetivo de evitar el control monopólico de los medios de comunicación y con la búsqueda de sistemas de subsidios gubernamentales para la colocación de anuncios publicitarios.

En tercer lugar, desde el punto de vista de los actores que intervienen en los medios de comunicación, la OG 34 toma un criterio amplio, incluyendo a comunicadores como bloggers y otros que se dedican a las publicaciones por intermedio de sitios web ${ }^{30}$. El Comité contempla los avances en materia de comunicación y nuevas tecnologías y descarta sistemas de colegiación o registro, salvo para generar accesos especiales. Se abordan también las restricciones indirectas a la libertad de expresión y el efecto perjudicial de la persecución penal por expresiones falsas o erradas ${ }^{31}$. Sin embargo, las leyes de desacato no son prohibidas, sino que se les exige un especial cuidado ${ }^{32}$. En cambio, el Comité sí se refiere expresamente a la despenalización de la difamación ${ }^{33}$. Esto demuestra una postura que rechaza la vía judicial como una opción eficiente para responder por aquellas expresiones lesivas, pero el Comité tampoco se arriesga a proponer soluciones alternativas como el derecho a rectificación y respuesta. Finalmente hay dos menciones importantes: el cuidado que debe tenerse en los delitos que repudian el terrorismo y la incompatibilidad declarada de aquellas leyes que penalizan las expresiones sobre hechos históricos ${ }^{34}$.

\section{Conclusiones}

En primer lugar debemos reconocer que, más que un desarrollo vanguardista, la Observación General No. 34 actualiza y reafirma en el sistema universal de protección de los derechos humanos algunos criterios que ya son reconocidos y aplicados en los sistemas regionales, principalmente en el sistema interamericano; donde por ejemplo, la Relatoría Especial para la Libertad de Expresión ha venido recogiendo en sus copiosos informes los criterios de "discurso protegido", "especial protección del debate político", "pluralidad de medios", entre otros.

Desde un punto de vista crítico, nos parece que la Observación opta por la postura de no definir en exceso los conceptos, ante el temor de que esto pudiese cerrar el espacio de aplicación del artículo 19. Así sucede en la delimitación del significado lingüístico de términos como "leyes", "orden público", "moral pública" y "seguridad nacional". Si a esta situación le sumamos la cautela con la que el Comité limita la extensión de algunas formas de restricciones especialmente lesivas

\footnotetext{
28 Comité de Derechos Humanos. Observación General No. 34, op. cit., párr. 38.

29 Ibídem, párr. 39.

30 Ibídem, párr. 44.

31 Ibídem, párr. 45.

32 La Observación General se vuelve más estricta cuando las expresiones se refieren a figuras públicas, allí se debe tender a la despenalización o a no tratar como ilegal publicaciones erradas o falsas, pero sin malicia.

33 Comité de Derechos Humanos. Observación General No. 34, op. cit., párr. 47.

34 Ibídem, párr. 50.
} 
como la penalización a través de las leyes de desacato, nos queda una sensación de escasez. Sin embargo, recordemos que a nivel universal las realidades jurídicas y políticas de los Estados son muy distintas, teniendo algunos de ellos un grado de desarrollo marcadamente menor.

Es importante destacar que el Comité ha reafirmado su reconocimiento acerca de las expresiones que rescatan o interpretan, con acierto o no, los hechos históricos; teniendo en cuenta su posible interacción con el derecho a la verdad o la comprensión histórica. Tanto en los precedentes de Francia como de Hungría ${ }^{35}$ se habían discutido este tipo de expresiones, pero siempre referido al caso concreto y vinculado a otros derechos; por lo que traerlo a una declaración general como es esta Observación le otorga una mayor proyección y así se acopla al desarrollo de otros ordenamientos jurídicos internacionales como el interamericano ${ }^{36}$.

Finalmente, quizás tengamos que esperar que el Comité comience a explayarse interpretando esta Observación General para conocer la incidencia de algunos aspectos particularmente prometedores como las prohibiciones de limitar el derecho de expresión e información con motivo de perseguir y penar los delitos de terrorismo y conexos, o el correlativo deber del Estado de informar al público sobre estos asuntos y darles participación en la difusión de la información, en relación con la obligación de proactivamente poner en dominio público la información de interés público.

Recibido: 31 octubre 2011

Aceptado: 31 enero 2012

35 Ver Comité de Derechos Humanos. Faurisson vs. Francia. Comunicación No. 550/93, Dictamen de 8 de noviembre de 1996. CCPR/C/58/D/550/1993 (1996); y Comité de Derechos Humanos. Observaciones finales sobre Hungría en el examen de los informes presentados por los Estados partes en virtud del artículo 40 del Pacto. 27 de octubre de 2010. $\mathrm{CCPR/C/HUN/CO/5,} \mathrm{párr.} 19$.

36 Ver Corte IDH. Caso Kimel vs. Argentina. Fondo, Reparaciones y Costas. Sentencia del 2 de mayo de 2008. Serie C No. 177 y Corte IDH. Caso Gomes Lund y otros (Guerrilha do Araguaia), op. cit. 
